\title{
Energy fluctuations in the homogeneous cooling state of granular gases
}

\author{
J. Javier Brey, M. I. García de Soria, P. Maynar, and M. J. Ruiz-Montero \\ Física Teórica, Universidad de Sevilla, Apdo. de Correos 1065, E-41080 Sevilla, Spain
}

(Received 25 March 2004; published 29 July 2004)

\begin{abstract}
Starting from the hierarchy of equations for microscopic densities in phase space, a general theory for fluctuations and correlations in a dilute granular gas of hard particles is developed. Then, the particular case of the homogeneous cooling state is addressed. Explicit expressions for some distributions describing the presence of velocity correlations and their dynamics are obtained. These correlations are inherent to the dissipative dynamics of the collisions. The implications for the behavior of the total energy of the system are analyzed and the results are expressed in terms of a fluctuation-dissipation theorem. The theoretical predictions are shown to be in agreement with results obtained by molecular dynamics simulations, which also indicate that energy fluctuations are well described by a Gaussian distribution.
\end{abstract}

DOI: 10.1103/PhysRevE.70.011302

PACS number(s): 45.70.-n, 51.10.+y, 05.20.Dd

\section{INTRODUCTION}

The understanding of the microscopic and macroscopic physical mechanisms involved in rapid granular flows has experimented a great advance in the last years [1,2]. Kinetic theory and nonequilibrium statistical mechanics methods have been extended to include inelasticity of collisions, which is the main feature of grain interactions and is associated with their macroscopic character. In these studies, the most widely employed idealized model for granular fluids is a system of smooth hard spheres (or disks in two dimensions) whose collisions are characterized by a constant coefficient of normal restitution. This coefficient measures the decrease in magnitude of the normal component of the relative velocity of the two colliding particles.

Starting from the dynamics of the particles, it is possible to derive the corresponding Liouville equation providing the basis for the application of many-body methods [3,4]. In particular, the inelastic Boltzmann equation describing the time evolution of the one-body distribution function is obtained in the dilute limit. This kinetic equation has been extensively used to address many fundamental issues and to compute averages of dynamical properties. Primary among these are the hydrodynamic equations, with explicit expressions for the transport coefficients, which have been derived by using a generalization of the Chapman-Enskog algorithm $[5,6]$. More recently, the hydrodynamic description has been identified in the context of the eigenfunctions and eigenvalues of the linearized inelastic Boltzmann collision operator $[7,8]$. To put these results in a proper context, it must be pointed out that there is no proof of time scale separation in rapid granular flows. At the level of the Boltzmann equation, this would require that the rest of the spectrum of the linearized operator is bounded away from the hydrodynamic modes, having a larger real part. This property has only been established for some simplified model Boltzmann collision operators $[8,9]$. On the other hand, granular hydrodynamics has been applied with success to many different situations, and the Chapmann-Enskog expressions for the transport coefficients have been confirmed by direct Monte Carlo simulations [10].

Most of the work carried out up to date has focused on kinetic equations for the one-body distribution and the infor- mation following from them, while the knowledge about fluctuations and correlations in granular gases is much more limited. First studies were based on mesoscopic fluctuating hydrodynamics [11] and also on model equations [12], and focused on the initial buildup of spatial correlations in the development of the clustering instability starting from an homogeneous system. Precollisional velocity correlations have been analyzed in detail at a fundamental level starting from the microscopic equations of motion and considering correlated sequences of collisions [13]. The system was initially in a state of thermal equilibrium and the results are valid at the short time and length scales. Then, although evidently relevant, they do not provide too much insight into the structure of the velocity correlations in any particular macroscopic state of the granular fluid.

The main goal of this paper is to formulate a theory of fluctuations for dilute granular gases and to apply it to one of the simplest possible situations, exploiting the scaling properties of the particular state under consideration. This will allow us to obtain explicit expressions for some distributions characterizing velocity fluctuations in the system. Special attention will be paid to the possibility of expressing the results in the form of a macroscopic theory of fluctuations, including fluctuation-dissipation relations. In order to develop a theory for equal and different time fluctuations, the hierarchy method will be used $[13,14]$. The general idea of the method is to derive from the Liouville equation hierarchies of coupled equations for the distribution functions describing the fluctuations. Then the hierarchies are closed by using the same kind of approximations as needed to derive the corresponding kinetic equation, in our case the Boltzmann equation. In this way, a unified formalism provides the usual kinetic equation as well as evolution equations for the one-time and two-time correlations.

An isolated fluid with elastic collisions tends to the equilibrium Gibbs state. The single particle distribution function is Maxwellian and there are no correlations between the velocities of the particles. Equilibrium fluctuations are well understood since long ago $[15,16]$. For inelastic collisions, there is no stationary state for an isolated system. The simplest state is the so-called "homogeneous cooling state" (HCS) with a monotonically decreasing temperature. Of 
course, this is a consequence of the dissipation of energy in collisions. For the same reason, the single particle distribution function deviates from the Maxwellian, both at small and large velocities [17-19]. Here, we will investigate a third implication of the dissipative character of collisions: the presence of velocity correlations, even in the low density limit. It must be realized that the existence of velocity correlations does not invalidate by itself the Boltzmann equation, as long as they are small enough, since they are obtained within a self-consistent theoretical frame, expected to be valid in the low density limit.

The study of fluctuations in the HCS is of primary importance for the development of a general theory of fluctuations in granular systems. The HCS defines the reference state from which macroscopic hydrodynamic equations can be derived [5], thus playing in this context a similar role to the equilibrium state in molecular systems. In the same way, a general theory of hydrodynamic fluctuations requires, as a necessary first step, the knowledge of the fluctuations and correlations occurring in the HCS. It should be stressed that no conceptual difficulty arises from using the HCS, which is a time-dependent state, as a reference state. In fact, the reference state is determined by the kinetic equation itself and cannot be arbitrarily chosen [20].

In the next section, hierarchies of equations for the onetime and two-time reduced distribution and correlation functions are derived. The closure relations obtained in the low density limit, leading in particular to the Boltzmann equation, are also discussed. The specific case of the HCS is considered in Sec. III, where the scaling property of this state is used to simplify the equations and eliminate trivial time dependencies. With an appropriate change of variables, the equations looks like corresponding to a stationary, but nonGibbs, state. In Secs. IV and V, marginal distributions for the one-time and two-time velocity correlations are introduced and their hydrodynamic parts are evaluated. The derived expressions are then used to obtain the second moment of the total energy fluctuations and the two-time energy correlation function. The theoretical predictions are compared with molecular dynamics simulation results and a quite good agreement is found over a wide range of values of the restitution coefficient. Moreover, the simulation data show that the scaled energy fluctuations are Gaussian within the numerical errors. Finally, Sec. VI contains a summary of the main results, and also a discussion of their connection with a fluctuation-dissipation relation.

\section{EVOLUTION EQUATIONS FOR THE REDUCED DISTRIBUTION FUNCTIONS}

We consider a dilute gas of smooth inelastic hard spheres $(d=3)$ or disks $(d=2)$ of mass $m$ and diameter $\sigma$. Let $X_{j}(t)$ $\equiv\left\{\boldsymbol{R}_{j}(t), \boldsymbol{V}_{j}(t)\right\}$ denote the position and velocity of particle $j$ at time $t$. Both $\boldsymbol{R}_{j}(t)$ and $\boldsymbol{V}_{j}(t)$ are parametric functions of the initial positions and velocities of all particles. For $t>0$ the time evolution of a phase function $A\left[\left\{X_{j}(t)\right\}\right]$ is defined by $[3,4]$

$$
A[\Gamma(t)]=e^{t \mathcal{L}(\Gamma)} A[\Gamma(0)],
$$

where $\Gamma \equiv\left\{X_{j} ; j=1, \ldots, N\right\}$ denotes the $N$-particle phase point. The generator of the dynamics $\mathcal{L}$ is

$$
\mathcal{L}(\Gamma)=L^{(0)}(\Gamma)+\frac{1}{2} \sum_{j}^{N} \sum_{i \neq j}^{N} T\left(X_{i}, X_{j}\right),
$$

with the first term generating free streaming,

$$
L^{(0)}=\sum_{j=1}^{N} \boldsymbol{V}_{j} \cdot \frac{\partial}{\partial \boldsymbol{R}_{j}},
$$

and the second terms describing velocity changes in collisions,

$$
\begin{aligned}
T\left(X_{i}, X_{j}\right)= & \sigma^{d-1} \int d \hat{\boldsymbol{\sigma}} \Theta\left(-\boldsymbol{V}_{i j} \cdot \hat{\boldsymbol{\sigma}}\right)\left|\boldsymbol{V}_{i j} \cdot \hat{\boldsymbol{\sigma}}\right| \delta\left(\boldsymbol{R}_{i j}-\boldsymbol{\sigma}\right) \\
& \times\left[b_{\boldsymbol{\sigma}}\left(\boldsymbol{V}_{i}, \boldsymbol{V}_{j}\right)-1\right] .
\end{aligned}
$$

Here $\boldsymbol{\sigma}=\sigma \hat{\boldsymbol{\sigma}}, d \hat{\boldsymbol{\sigma}}$ is the solid angle element for $\hat{\boldsymbol{\sigma}}, \boldsymbol{R}_{i j} \equiv \boldsymbol{R}_{i}$ $-\boldsymbol{R}_{j}, \boldsymbol{V}_{i j} \equiv \boldsymbol{V}_{i}-\boldsymbol{V}_{j}, \Theta$ is the Heaviside step function, and the operator $b_{\boldsymbol{\sigma}}\left(\boldsymbol{V}_{i}, \boldsymbol{V}_{j}\right)$ changes functions of $\boldsymbol{V}_{i}, \boldsymbol{V}_{j}$ to the same functions of the scattered velocities given by

$$
\begin{gathered}
\boldsymbol{V}_{i} \rightarrow \boldsymbol{V}_{i}^{\prime} \equiv b_{\boldsymbol{\sigma}} \boldsymbol{V}_{i}=\boldsymbol{V}_{i}-\frac{1+\alpha}{2}\left(\hat{\boldsymbol{\sigma}} \cdot \boldsymbol{V}_{i j}\right) \hat{\boldsymbol{\sigma}}, \\
\boldsymbol{V}_{j} \rightarrow \boldsymbol{V}_{j}^{\prime} \equiv b_{\boldsymbol{\sigma}} \boldsymbol{V}_{j}=\boldsymbol{V}_{j}+\frac{1+\alpha}{2}\left(\hat{\boldsymbol{\sigma}} \cdot \boldsymbol{V}_{i j}\right) \hat{\boldsymbol{\sigma}} .
\end{gathered}
$$

Inelasticity of collisions is characterized by means of the coefficient of normal restitution $\alpha$ taking values in the interval $0<\alpha \leqslant 1$.

Microscopic densities in phase space $F_{s}\left(x_{1}, x_{2}, \ldots, x_{s}, t\right)$ are defined by

$$
\begin{gathered}
F_{1}\left(x_{1}, t\right)=\sum_{j=1}^{N} \delta\left(x_{1}-X_{j}(t)\right), \\
F_{2}\left(x_{1}, x_{2}, t\right)=\sum_{i}^{N} \sum_{j \neq i}^{N} \delta\left(x_{1}-X_{i}(t)\right) \delta\left(x_{2}-X_{j}(t)\right),
\end{gathered}
$$

etc. The lower-case variables $x_{i} \equiv\left\{\boldsymbol{r}_{i}, \boldsymbol{v}_{i}\right\}$ are field variables referring to a particular point in $\mu$ space. A hierarchy of equations for the above densities is obtained from Eq. (1). The first two equations, valid for $t>0$, are

$$
\begin{gathered}
{\left[\frac{\partial}{\partial t}+L^{(0)}\left(x_{1}\right)\right] F_{1}\left(x_{1}, t\right)=\int d x_{2} \bar{T}\left(x_{1}, x_{2}\right) F_{2}\left(x_{1}, x_{2}, t\right),} \\
{\left[\frac{\partial}{\partial t}+L^{(0)}\left(x_{1}, x_{2}\right)\right] F_{2}\left(x_{1}, x_{2}, t\right)=\bar{T}\left(x_{1}, x_{2}\right) F_{2}\left(x_{1}, x_{2}, t\right)} \\
+\int d x_{3}\left[\bar{T}\left(x_{1}, x_{3}\right)+\bar{T}\left(x_{2}, x_{3}\right)\right] F_{3}\left(x_{1}, x_{2}, x_{3}, t\right),
\end{gathered}
$$

where

$$
L^{(0)}\left(x_{1}, x_{2}\right)=L^{(0)}\left(x_{1}\right)+L^{(0)}\left(x_{2}\right), \quad L^{(0)}\left(x_{i}\right)=\boldsymbol{v}_{i} \cdot \frac{\partial}{\partial \boldsymbol{r}_{i}},
$$

and $\bar{T}\left(x_{i}, x_{j}\right)$ is the binary collision operator 


$$
\begin{aligned}
\bar{T}\left(x_{i}, x_{j}\right)= & \sigma^{d-1} \int d \hat{\boldsymbol{\sigma}} \Theta\left(\boldsymbol{v}_{i j} \cdot \hat{\boldsymbol{\sigma}}\right)\left|\boldsymbol{v}_{i j} \cdot \hat{\boldsymbol{\sigma}}\right| \\
& \times\left[\alpha^{-2} \delta\left(\boldsymbol{r}_{i j}-\boldsymbol{\sigma}\right) b_{\boldsymbol{\sigma}}^{-1}\left(\boldsymbol{v}_{i}, \boldsymbol{v}_{j}\right)-\delta\left(\boldsymbol{r}_{i j}+\boldsymbol{\sigma}\right)\right] .
\end{aligned}
$$

The operator $b_{\boldsymbol{\sigma}}^{-1}\left(\boldsymbol{v}_{i}, \boldsymbol{v}_{j}\right)$ replaces all the velocities $\boldsymbol{v}_{i}$ and $\boldsymbol{v}_{j}$ appearing to its right by the precollisional values $\boldsymbol{v}_{1}^{*}$ and $\boldsymbol{v}_{2}^{*}$,

$$
\begin{gathered}
\boldsymbol{v}_{i} \rightarrow \boldsymbol{v}_{i}^{*} \equiv b_{\boldsymbol{\sigma}}^{-1} \boldsymbol{v}_{i}=\boldsymbol{v}_{i}-\frac{1+\alpha}{2 \alpha}\left(\hat{\boldsymbol{\sigma}} \cdot \boldsymbol{v}_{i j}\right) \hat{\boldsymbol{\sigma}}, \\
\boldsymbol{v}_{j} \rightarrow \boldsymbol{v}_{j}^{*} \equiv b_{\boldsymbol{\sigma}}^{-1} \boldsymbol{v}_{j}=\boldsymbol{v}_{j}+\frac{1+\alpha}{2 \alpha}\left(\hat{\boldsymbol{\sigma}} \cdot \boldsymbol{v}_{i j}\right) \hat{\boldsymbol{\sigma}} .
\end{gathered}
$$

The averages of the microscopic densities $F_{s}\left(x_{1}, x_{2}, \cdots, x_{s}, t\right)$ over the probability distribution function $\rho(\Gamma, 0)$ characterizing the initial state of the system are the usual one-time reduced distribution functions,

$$
f_{s}\left(x_{1}, \ldots, x_{s}, t\right) \equiv\left\langle F_{s}\left(x_{1}, \cdots, x_{s}, t\right)\right\rangle,
$$

where we have introduced the notation

$$
\langle G\rangle \equiv \int d \Gamma G(\Gamma) \rho(\Gamma, 0) .
$$

The one-time reduced distributions obey a hierarchy of equations that follow directly by averaging the hierarchy for the microscopic densities. This is the generalization for inelastic collisions of the BBGKY hierarchy. In particular, from Eqs. (8) and (9) it is trivially obtained [3]:

$$
\begin{gathered}
{\left[\frac{\partial}{\partial t}+L^{(0)}\left(x_{1}\right)\right] f_{1}\left(x_{1}, t\right)=\int d x_{2} \bar{T}\left(x_{1}, x_{2}\right) f_{2}\left(x_{1}, x_{2}, t\right),} \\
{\left[\frac{\partial}{\partial t}+L^{(0)}\left(x_{1}, x_{2}\right)\right] f_{2}\left(x_{1}, x_{2}, t\right)=\bar{T}\left(x_{1}, x_{2}\right) f_{2}\left(x_{1}, x_{2}, t\right)} \\
+\int d x_{3}\left[\bar{T}\left(x_{1}, x_{3}\right)+\bar{T}\left(x_{2}, x_{3}\right)\right] f_{3}\left(x_{1}, x_{2}, x_{3}, t\right) .
\end{gathered}
$$

Two-time reduced distribution functions can also be defined in terms of the microscopic densities as

$$
\begin{aligned}
f_{r, s}\left(x_{1}, \ldots, x_{r}, t ; x_{1}^{\prime}, \ldots, x_{s}^{\prime}, t^{\prime}\right) \equiv & \left\langle F_{r}\left(x_{1}, \ldots, x_{r}, t\right)\right. \\
& \left.\times F_{s}\left(x_{1}^{\prime}, \ldots, x_{s}^{\prime}, t^{\prime}\right)\right\rangle .
\end{aligned}
$$

It will be assumed that $t>t^{\prime}>0$ for concreteness. Again, evolution equations for these quantities are derived from the hierarchy for the densities $F_{r}$. The lowest order distribution, $f_{1,1}$, is seen to obey the equation

$$
\begin{aligned}
& {\left[\frac{\partial}{\partial t}+L^{(0)}\left(x_{1}\right)\right] f_{1,1}\left(x_{1}, t ; x_{1}^{\prime}, t^{\prime}\right)} \\
& =\int d x_{2} \bar{T}\left(x_{1}, x_{2}\right) f_{2,1}\left(x_{1}, x_{2}, t ; x_{1}^{\prime}, t^{\prime}\right),
\end{aligned}
$$

which has to be solved with the initial condition

$$
f_{1,1}\left(x_{1}, t^{\prime} ; x_{1}^{\prime}, t^{\prime}\right)=\delta\left(x_{1}-x_{1}^{\prime}\right) f\left(x_{1}, t^{\prime}\right)+f_{2}\left(x_{1}, x_{2}, t^{\prime}\right),
$$

that follows directly from the definition in Eq. (17). With regard to the above equations for the reduced distribution functions, it must be reminded that although the streaming operators also generate unphysical trajectories for unphysical initial conditions, the latter have a vanishing weight in the initial probability distribution and, therefore, the dynamics in all the equations for the reduced distribution functions is in fact well behaved.

It is convenient to introduce also correlation functions through the usual cluster expansions. From the one-time reduced distributions, one-time correlations $g_{s}\left(x_{1}, \ldots, x_{s}, t\right)$ are defined by

$$
\begin{aligned}
& f_{2}\left(x_{1}, x_{2}, t\right)=f_{1}\left(x_{1}, t\right) f_{1}\left(x_{2}, t\right)+g_{2}\left(x_{1}, x_{2}, t\right), \\
& f_{3}\left(x_{1}, x_{2}, x_{3}, t\right)= f_{1}\left(x_{1}, t\right) f_{1}\left(x_{2}, t\right) f_{1}\left(x_{3}, t\right)+f_{1}\left(x_{1}, t\right) g_{2}\left(x_{2}, x_{3}, t\right) \\
&+f_{1}\left(x_{2}, t\right) g_{2}\left(x_{1}, x_{3}, t\right)+f_{1}\left(x_{3}, t\right) g_{2}\left(x_{1}, x_{2}, t\right) \\
&+g_{3}\left(x_{1}, x_{2}, x_{3}, t\right),
\end{aligned}
$$

etc. Similarly, two-time correlation functions $h_{r, s}$ can be defined. In particular, $h_{1,1}$ and $h_{2,1}$ are introduced through

$$
f_{1,1}\left(x_{1}, t ; x_{1}^{\prime}, t^{\prime}\right)=f_{1}\left(x_{1}, t\right) f_{1}\left(x_{1}^{\prime}, t^{\prime}\right)+h_{1,1}\left(x_{1}, t ; x_{1}^{\prime}, t^{\prime}\right),
$$

$$
\begin{aligned}
f_{2,1}\left(x_{1}, x_{2}, t ; x_{1}^{\prime}, t^{\prime}\right)= & f_{1}\left(x_{1}, t\right) f_{1}\left(x_{2}, t\right) f_{1}\left(x_{1}^{\prime}, t^{\prime}\right) \\
& +g_{2}\left(x_{1}, x_{2}, t\right) f_{1}\left(x_{1}^{\prime}, t^{\prime}\right) \\
& +h_{1,1}\left(x_{1}, t ; x_{1}^{\prime}, t^{\prime}\right) f_{1}\left(x_{2}, t\right) \\
& +h_{1,1}\left(x_{2}, t ; x_{1}^{\prime}, t^{\prime}\right) f_{1}\left(x_{1}, t\right) \\
& +h_{2,1}\left(x_{1}, x_{2}, t ; x_{1}^{\prime}, t^{\prime}\right) .
\end{aligned}
$$

Evolution equations for the correlation functions are obtained from the equations for the corresponding reduced distributions. Thus from Eqs. (15) and (16) one gets

$$
\begin{aligned}
& {\left[\frac{\partial}{\partial t}+L^{(0)}\left(x_{1}\right)\right] f_{1}\left(x_{1}, t\right)=} \int d x_{2} \bar{T}\left(x_{1}, x_{2}\right)\left[f_{1}\left(x_{1}, t\right) f_{1}\left(x_{2}, t\right)\right. \\
&\left.+g_{2}\left(x_{1}, x_{2}, t\right)\right] \\
& {\left[\frac{\partial}{\partial t}+\right.}\left.L^{(0)}\left(x_{1}, x_{2}\right)\right] g_{2}\left(x_{1}, x_{2}, t\right) \\
&= \bar{T}\left(x_{1}, x_{2}\right)\left[f_{1}\left(x_{1}, t\right) f_{1}\left(x_{2}, t\right)+g_{2}\left(x_{1}, x_{2}, t\right)\right] \\
& \quad+\int d x_{3}\left(1+P_{12}\right) \bar{T}\left(x_{1}, x_{3}\right)\left[g_{2}\left(x_{1}, x_{2}, t\right) f_{1}\left(x_{3}, t\right)\right. \\
&\left.\quad+g_{2}\left(x_{2}, x_{3}, t\right) f_{1}\left(x_{1}, t\right)+g_{3}\left(x_{1}, x_{2}, x_{3}, t\right)\right]
\end{aligned}
$$

where the permutation operator $P_{12}$ interchanges the labels of particles 1 and 2 in the quantities to its right. In an analogous way, from Eq. (18) it results 


$$
\begin{aligned}
{\left[\frac{\partial}{\partial t}+\right.} & \left.L^{(0)}\left(x_{1}\right)\right] h_{1,1}\left(x_{1}, t ; x_{1}^{\prime}, t^{\prime}\right) \\
= & \int d x_{2} \bar{T}\left(x_{1}, x_{2}\right)\left[h_{1,1}\left(x_{1}, t ; x_{1}^{\prime}, t^{\prime}\right) f_{1}\left(x_{2}, t\right)\right. \\
& \left.+h_{1,1}\left(x_{2}, t ; x_{1}^{\prime}, t^{\prime}\right) f_{1}\left(x_{1}, t\right)+h_{2,1}\left(x_{1}, x_{2}, t ; x_{1}^{\prime}, t^{\prime}\right)\right] .
\end{aligned}
$$

The last equation holds for $t>t^{\prime}$ and the initial condition is

$$
h_{1,1}\left(x_{1}, t^{\prime} ; x_{1}^{\prime}, t^{\prime}\right)=g_{2}\left(x_{1}, x_{1}^{\prime}, t^{\prime}\right)+\delta\left(x_{1}-x_{1}^{\prime}\right) f_{1}\left(x_{1}, t^{\prime}\right) .
$$

All the equations derived in this section are exact consequences of the dynamics of the particles as defined by the generator given in Eq. (2). In fact, they are the extension to inelastic hard particles of the equations discussed in Ref. [14]. In the next sections, the low density limit and the particular case of the HCS will be addressed.

\section{LOW DENSITY FLUCTUATIONS AROUND THE HOMOGENEOUS COOLING STATE}

In order to obtain closed kinetic equations for the reduced distribution functions, some kind of closure relations for the hierarchies of equations derived in the previous section are required. Here we are interested in the limit of a dilute inelastic gas and the method we will apply is based on the general assumption that the order in the density of correlation functions increases as the number of involved particles increases. More precisely, what it is assumed is that the above is true for the precollisional part of the correlation functions when two of the particles are at contact [21]. This method was discussed in detail by Ernst and Cohen for the hard sphere gas with elastic collisions [14] and it has been already applied to the study of one-time reduced distributions for inelastic hard particles [4,22]. Closely related results follow from a formal small parameter expansion of the reduced distribution functions [20,23]. From a physical point of view, the underlying idea of these kinetic theories is that no hypothesis are necessary to derive equations for fluctuations and time-correlations beyond those required to derive the kinetic equation for the one-particle distribution function, i.e., the Boltzmann equation in the dilute gas. Let us stress that this seems to be a quite general property, beyond the range of validity of the Boltzmann equation, as emphasized in Ref. [24].

Then, in the low density limit, the term involving $\bar{T}\left(x_{1}, x_{2}\right) g_{2}\left(x_{1}, x_{2}, t\right)$ in Eq. (24) is assumed to be negligible as compared with the one involving $\bar{T}\left(x_{1}, x_{2}\right) f_{1}\left(x_{1}, t\right) f_{1}\left(x_{2}, t\right)$. Furthermore, in the same limit the spatial separation between the centers of colliding particles can be neglected, so that the above mentioned equation reduces to

$$
\left[\frac{\partial}{\partial t}+L^{(0)}\left(x_{1}\right)\right] f_{1}\left(x_{1}, t\right)=J\left[x_{1}, t \mid f_{1}\right]
$$

where

$$
J\left[x_{1}, t \mid f_{1}\right]=\int d x_{2} \delta\left(\boldsymbol{r}_{12}\right) \bar{T}_{0}\left(\boldsymbol{v}_{1}, \boldsymbol{v}_{2}\right) f_{1}\left(x_{1}, t\right) f_{1}\left(x_{2}, t\right),
$$

$$
\begin{aligned}
\bar{T}_{0}\left(\boldsymbol{v}_{1}, \boldsymbol{v}_{2}\right)= & \sigma^{d-1} \int d \hat{\boldsymbol{\sigma}} \Theta\left(\boldsymbol{v}_{12} \cdot \hat{\boldsymbol{\sigma}}\right)\left|\boldsymbol{v}_{12} \cdot \hat{\boldsymbol{\sigma}}\right| \\
& \times\left[\alpha^{-2} b_{\boldsymbol{\sigma}}^{-1}\left(\boldsymbol{v}_{1}, \boldsymbol{v}_{2}\right)-1\right] .
\end{aligned}
$$

This is the Boltzmann equation for smooth inelastic hard spheres [3,17]. Similarly, Eq. (25) reduces to

$$
\begin{aligned}
& {\left[\frac{\partial}{\partial t}+L^{(0)}\left(x_{1}, x_{2}\right)-K\left[x_{1}, t \mid f_{1}\right]-K\left[x_{2}, t \mid f_{1}\right]\right] g_{2}\left(x_{1}, x_{2}, t\right)} \\
& \quad=\delta\left(\mathbf{r}_{12}\right) \bar{T}_{0}\left(\boldsymbol{v}_{1}, \boldsymbol{v}_{2}\right) f_{1}\left(x_{1}, t\right) f_{1}\left(x_{2}, t\right),
\end{aligned}
$$

where we have introduced the operator

$$
K\left[x_{i}, t \mid f_{1}\right] \equiv \int d x_{3} \delta\left(\boldsymbol{r}_{i 3}\right) \bar{T}_{0}\left(\boldsymbol{v}_{i}, \boldsymbol{v}_{3}\right)\left(1+P_{i 3}\right) f_{1}\left(x_{3}, t\right) .
$$

Therefore, two particle correlations are generated from the uncorrelated product of solutions of the Boltzmann equation through inelastic binary collisions. Of course, the solutions of this equation must be consistent with the approximation leading to the Boltzmann equation. The low density limit of the equation for the two-time correlations, Eq. (26), follows by neglecting the term proportional to $h_{2,1}$ on the right-hand side and also the distance between colliding particles, so that it is given by

$$
\left[\frac{\partial}{\partial t}+L^{(0)}\left(x_{1}\right)-K\left[x_{1}, t \mid f_{1}\right]\right] h_{1,1}\left(x_{1}, t, x_{1}^{\prime}, t^{\prime}\right)=0 .
$$

Finally, an equation for $h_{1,1}\left(x_{1}, t ; x_{1}^{\prime}, t\right)$ is obtained by taking time derivative in Eq. (27) and using Eqs. (28) and (31),

$$
\begin{aligned}
& {\left[\frac{\partial}{\partial t}+L^{(0)}\left(x_{1}, x_{1}^{\prime}\right)-K\left[x_{1}, t \mid f_{1}\right]-K\left[x_{1}^{\prime}, t \mid f_{1}\right]\right] h_{1,1}\left(x_{1}, t ; x_{1}^{\prime}, t\right)} \\
& \quad=\Gamma\left(x_{1}, x_{1}^{\prime}, t\right),
\end{aligned}
$$

with

$$
\begin{aligned}
\Gamma\left(x_{1}, x_{1}^{\prime}, t\right)= & \delta\left(x_{1}-x_{1}^{\prime}\right) J\left[x_{1}, t \mid f_{1}\right]-\left(K\left[x_{1}, t \mid f_{1}\right]\right. \\
& \left.+K\left[x_{1}^{\prime}, t \mid f_{1}\right]\right) \delta\left(x_{1}-x_{1}^{\prime}\right) f_{1}\left(x_{1}, t\right) \\
& +\delta\left(\boldsymbol{r}_{1}-\boldsymbol{r}_{1}^{\prime}\right) \bar{T}_{0}\left(\boldsymbol{v}_{1}, \boldsymbol{v}_{1}^{\prime}\right) f_{1}\left(x_{1}, t\right) f_{1}\left(x_{1}^{\prime}, t\right) .
\end{aligned}
$$

It is worth pointing out that the above derivation makes no reference to whether collisions are elastic or inelastic. In fact, when it is compared with the analysis carried out in Ref. [14], the only difference is in the replacement of the elastic binary collision operators by the inelastic ones and this does not affect the density dependence of each of the contributions to the equations. This is also the case when the kinetic equations are obtained from a formal expansion in a dimensionless density parameter $[20,23]$. Therefore, at least at a superficial level, it seems that the theoretical basis for low density kinetic theory is the same both for elastic and inelastic systems. Of course, the actual range of validity of the kinetic equations, i.e., how small the density of the system must be so that the above kinetic equations provide an accurate description, might depend on the inelasticity of the system, and also on the particular state being considered. 
The inelastic Boltzmann equation (28) admits a special solution $f_{\mathrm{HCS}}(\mathbf{v}, t)$ describing the HCS for which all the time dependence of the distribution function occurs through the granular temperature (defined from the average kinetic energy). This solution has the scaled form [17]

$$
f_{\mathrm{HCS}}(\boldsymbol{v}, t)=n_{H} v_{0}^{-d}(t) \chi_{\mathrm{HCS}}(\boldsymbol{c}) .
$$

Here $n_{H}$ is the uniform number of particles density,

$$
v_{0}(t) \equiv\left[\frac{2 k_{B} T_{\mathrm{HCS}}(t)}{m}\right]^{1 / 2},
$$

$k_{B}$ being the Boltzmann constant, and $\chi_{\mathrm{HCS}}(\boldsymbol{c})$ an isotropic function of scaled velocity $\boldsymbol{c}=\boldsymbol{v} / v_{0}(t)$. The above scaling property implies that the temperature obeys the equation

$$
\frac{\partial T_{\mathrm{HCS}}(t)}{\partial t}+\zeta_{\mathrm{HCS}}(t) T_{\mathrm{HCS}}(t)=0 .
$$

The cooling rate $\zeta_{\mathrm{HCS}}(t)$ is a given bilinear functional of $f_{\mathrm{HCS}}$, and it is proportional to $T_{\mathrm{HCS}}(t)^{1 / 2}$. Its explicit expression will not be given here [7,17]. Substitution of Eq. (36) into the Boltzmann equation (28) and use of Eq. (38) leads to a closed equation for $\chi_{\mathrm{HCS}}(\boldsymbol{c})$,

$$
\frac{\zeta_{0}}{2} \frac{\partial}{\partial c} \cdot\left(\boldsymbol{c} \chi_{\mathrm{HCS}}\right)=J_{c}\left[\boldsymbol{c} \mid \chi_{\mathrm{HCS}}\right],
$$

where

$$
\begin{aligned}
\zeta_{0} & =\frac{\ell \zeta_{\mathrm{HCS}}(t)}{v_{0}(t)} \\
& =\frac{\left(1-\alpha^{2}\right) \pi^{(d-1) / 2}}{2 \Gamma\left(\frac{d+3}{2}\right) d} \int d c_{1} \int d c_{2} c_{12}^{3} \chi_{\mathrm{HCS}}\left(c_{1}\right) \chi_{\mathrm{HCS}}\left(\boldsymbol{c}_{2}\right)
\end{aligned}
$$

is the time-independent dimensionless cooling rate of the HCS and

$$
J_{c}\left[\boldsymbol{c} \mid \chi_{\mathrm{HCS}}\right]=\int d \boldsymbol{c}_{1} \bar{T}_{0}\left(\boldsymbol{c}, \boldsymbol{c}_{1}\right) \chi_{\mathrm{HCS}}(\boldsymbol{c}) \chi_{\mathrm{HCS}}\left(\boldsymbol{c}_{1}\right),
$$

$\bar{T}_{0}\left(\boldsymbol{c}, \boldsymbol{c}_{1}\right)=\int d \hat{\boldsymbol{\sigma}} \Theta\left[\left(\boldsymbol{c}-\boldsymbol{c}_{1}\right) \cdot \hat{\boldsymbol{\sigma}}\right]\left(\boldsymbol{c}-\boldsymbol{c}_{1}\right) \cdot \hat{\boldsymbol{\sigma}}\left[\alpha^{-2} b_{\boldsymbol{\sigma}}^{-1}\left(\boldsymbol{c}, \boldsymbol{c}_{1}\right)-1\right]$.

The operator $b_{\boldsymbol{\sigma}}^{-1}\left(\boldsymbol{c}, \boldsymbol{c}_{1}\right)$ is again defined by Eqs. (12), but replacing the velocities $\boldsymbol{v}_{i}$ and $\boldsymbol{v}_{j}$ by $\boldsymbol{c}$ and $\boldsymbol{c}_{1}$, respectively.

It is useful to introduce dimensionless time and length scales by

$$
s=\int_{0}^{t} d t_{1} \frac{v_{0}\left(t_{1}\right)}{\ell}, \quad l=\ell^{-1} \boldsymbol{r},
$$

respectively, where $\ell \equiv\left(n_{H} \sigma^{d-1}\right)^{-1}$ is proportional to the mean free path of the gas. Thus, $s(t)$ is a measure of the average number of collisions per particle in the interval $(0, t)$. In terms of $s$, Eq. (38) becomes

$$
\frac{\partial T_{\mathrm{HCS}}}{\partial s}=-\zeta_{0} T_{\mathrm{HCS}}(s),
$$

showing that the temperature of the HCS decreases exponentially on this time scale. The equivalent expression in the original time scale is given by Haff's law [25],

$$
T_{\mathrm{HCS}}(t)=T_{\mathrm{HCS}}(0)\left[1+\frac{1}{2} \zeta_{\mathrm{HCS}}(0) t\right]^{-2} .
$$

In order to particularize the equations for the two-particle correlations, the concept of the HCS as introduced above will be extended. It will be assumed that in the HCS the $N$-particle distribution function of the system, $\rho_{\mathrm{HCS}}(\Gamma, t)$, is given by a scaling function for which all the time dependence occurs through the scaling of the velocities with the thermal velocity $v_{0}(t)[3]$,

$$
\rho_{\mathrm{HCS}}(\Gamma, t)=\left[v_{0}(t)\right]^{-d N} \rho_{\mathrm{HCS}}^{*}\left(\left\{\boldsymbol{r}_{i j}, \boldsymbol{c}_{i}\right\}\right) .
$$

The scaled distribution $\rho_{\mathrm{HCS}}^{*}$ is invariant under space translations. Therefore, $\rho_{\mathrm{HCS}}(\Gamma, t)$ represents a spatially homogeneous fluid. The above scaling implies a similar scaling for all the reduced distribution functions and, in particular, the one given by Eq. (36) for the one-particle distribution function. Then, Eq. (31) when applied to the HCS becomes

$$
\begin{aligned}
& {\left[\boldsymbol{c}_{12} \cdot \frac{\partial}{\partial \boldsymbol{l}_{12}}-\Lambda\left(\boldsymbol{c}_{1}\right)-\Lambda\left(\boldsymbol{c}_{2}\right)\right] \widetilde{g}_{\mathrm{HCS}}\left(\boldsymbol{l}_{12}, \boldsymbol{c}_{1}, \boldsymbol{c}_{2}\right)} \\
& \quad=\delta\left(\boldsymbol{l}_{12}\right) \bar{T}_{0}\left(\boldsymbol{c}_{1}, \boldsymbol{c}_{2}\right) \chi_{\mathrm{HCS}}\left(\boldsymbol{c}_{1}\right) \chi_{\mathrm{HCS}}\left(\boldsymbol{c}_{2}\right),
\end{aligned}
$$

where $\Lambda\left(\boldsymbol{c}_{i}\right)$ is the linearized Boltzmann collision operator in the scaled representation,

$$
\Lambda\left(\boldsymbol{c}_{i}\right)=\int d \boldsymbol{c}_{3} \bar{T}_{0}\left(\boldsymbol{c}_{i}, \boldsymbol{c}_{3}\right)\left(1+P_{i 3}\right) \chi_{\mathrm{HCS}}\left(\boldsymbol{c}_{3}\right)-\frac{\zeta_{0}}{2} \frac{\partial}{\partial \boldsymbol{c}_{i}} \cdot \boldsymbol{c}_{i},
$$

and the scaled two-particle one-time correlation function is defined by

$$
\widetilde{g}_{\mathrm{HCS}}\left(\boldsymbol{l}_{12}, \boldsymbol{c}_{1}, \boldsymbol{c}_{2}\right)=n_{H}^{-1} \ell^{d} v_{0}^{2 d}(t) g_{2, \mathrm{HCS}}\left(\boldsymbol{r}_{12}, \boldsymbol{v}_{1}, \boldsymbol{v}_{2}, t\right) .
$$

This quantity does not depend on $s$ as a consequence of the scaling property. In a similar way, Eq. (33) yields

$$
\left[\frac{\partial}{\partial s}+\boldsymbol{c}_{1} \cdot \frac{\partial}{\partial \boldsymbol{l}_{1}}-\Lambda\left(\boldsymbol{c}_{1}\right)\right] \tilde{h}_{\mathrm{HCS}}\left(\boldsymbol{l}_{1}-\boldsymbol{l}_{1}^{\prime}, \boldsymbol{c}_{1}, s-s^{\prime} ; \boldsymbol{c}_{1}^{\prime}\right)=0,
$$

with

$$
\begin{aligned}
\tilde{h}_{\mathrm{HCS}}\left(\boldsymbol{l}_{1}-\boldsymbol{l}_{1}^{\prime}, \boldsymbol{c}_{1}, s-s^{\prime} ; \boldsymbol{c}_{1}^{\prime}\right)= & n_{H}^{-1} \ell^{d} v_{0}^{d}(t) v_{0}^{d}\left(t^{\prime}\right) \\
& \times h_{1,1, \mathrm{HCS}}\left(x_{1}, t ; x_{1}^{\prime}, t^{\prime}\right) .
\end{aligned}
$$

The initial condition for this equation is

$$
\begin{aligned}
\widetilde{h}_{\mathrm{HCS}}\left(\boldsymbol{l}_{1}-\boldsymbol{l}_{1}^{\prime}, \boldsymbol{c}_{1}, 0 ; \boldsymbol{c}_{1}^{\prime}\right) \equiv & \widetilde{h}_{\mathrm{HCS}}\left(\boldsymbol{l}_{1}-\boldsymbol{l}_{1}^{\prime}, \boldsymbol{c}_{1} ; \boldsymbol{c}_{1}^{\prime}\right) \\
= & \widetilde{g}_{\mathrm{HCS}}\left(\boldsymbol{l}_{1}-\boldsymbol{l}_{1}^{\prime}, \boldsymbol{c}_{1}, \boldsymbol{c}_{1}^{\prime}\right) \\
& +\delta\left(\boldsymbol{c}_{1}-\boldsymbol{c}_{1}^{\prime}\right) \delta\left(\boldsymbol{l}_{1}-\boldsymbol{l}_{1}^{\prime}\right) \chi_{\mathrm{HCS}}\left(\boldsymbol{c}_{1}\right) .
\end{aligned}
$$


In the above expressions, we have made explicit the translational invariance of the HCS. Finally, Eq. (34) becomes

$$
\begin{gathered}
{\left[\boldsymbol{c}_{1} \cdot \frac{\partial}{\partial \boldsymbol{l}_{1}}+c_{1}^{\prime} \cdot \frac{\partial}{\partial \boldsymbol{l}_{1}^{\prime}}-\Lambda\left(\boldsymbol{c}_{1}\right)-\Lambda\left(\boldsymbol{c}_{1}^{\prime}\right)\right] \tilde{h}_{\mathrm{HCS}}\left(\boldsymbol{l}_{1}-\boldsymbol{l}_{1}^{\prime}, \boldsymbol{c}_{1} ; \boldsymbol{c}_{1}^{\prime}\right)} \\
=\delta\left(\boldsymbol{l}_{1}-\boldsymbol{l}_{1}^{\prime}\right) \widetilde{\Gamma}\left(\boldsymbol{c}_{1}, \boldsymbol{c}_{1}^{\prime}\right), \\
\widetilde{\Gamma}\left(\boldsymbol{c}_{1}, \boldsymbol{c}_{1}^{\prime}\right)=-\left[\Lambda\left(\boldsymbol{c}_{1}\right)+\Lambda\left(\boldsymbol{c}_{1}^{\prime}\right)\right] \delta\left(\boldsymbol{c}_{1}-\boldsymbol{c}_{1}^{\prime}\right) \chi_{\mathrm{HCS}}\left(\boldsymbol{c}_{1}\right) \\
+\bar{T}_{0}\left(\boldsymbol{c}_{1}, \boldsymbol{c}_{1}^{\prime}\right) \chi_{\mathrm{HCS}}\left(\boldsymbol{c}_{1}\right) \chi_{\mathrm{HCS}}\left(\boldsymbol{c}_{1}^{\prime}\right) .
\end{gathered}
$$

Equations (47) and (53) describe the correlations between equal time fluctuations. Their solutions are related through Eq. (52). In both equations, the distribution function of the HCS is needed and has to be found from Eq. (39). The same happens with Eq. (50) for the unequal time fluctuations. Nevertheless, the analytical form of $\chi_{\mathrm{HCS}}(\boldsymbol{c})$ is only partially known. In particular, an expansion about the Gaussian in Sonine polynomials has been used. To first order, $\chi_{\mathrm{HCS}}(\boldsymbol{c})$ is approximated by

$$
\chi_{\mathrm{HCS}}(\boldsymbol{c})=\frac{e^{-c^{2}}}{\pi^{d / 2}}\left[1+a_{2}(\alpha) S^{(2)}\left(c^{2}\right)\right]
$$

where

$$
S^{(2)}\left(c^{2}\right)=\frac{c^{4}}{2}-\frac{d+2}{2} c^{2}+\frac{d(d+2)}{8} .
$$

The coefficient $a_{2}$ is related with the fourth moment of $\chi_{\mathrm{HCS}}(\boldsymbol{c})$ by

$$
a_{2}(\alpha)=\frac{4\left\langle c^{4}\right\rangle}{d(d+2)}-1 ; \quad\left\langle c^{4}\right\rangle \equiv \int d c c^{4} \chi_{\mathrm{HCS}}(\boldsymbol{c}) .
$$

Substitution of Eq. (55) into Eq. (39) leads to a closed equation for the coefficient $a_{2}$, and by neglecting nonlinear terms in $a_{2}$ one gets $[17,18]$

$$
a_{2}(\alpha)=\frac{16(1-\alpha)\left(1-2 \alpha^{2}\right)}{9+24 d+(8 d-41) \alpha+30 \alpha^{2}-30 \alpha^{3}} .
$$

Using Eq. (55), the following approximate expression for the dimensionless cooling rate is found:

$$
\zeta_{0}=\frac{\sqrt{2} \pi^{(d-1) / 2}\left(1-\alpha^{2}\right)}{\Gamma\left(\frac{d}{2}\right) d}\left[1+\frac{3}{16} a_{2}(\alpha)\right] .
$$

The above expressions have been shown to agree with numerical results obtained by means of the direct simulation Monte Carlo method in the thermal velocity region, i.e. for velocities $c$ of the order of a few units [26]. Let us mention that the shape of the scaling distribution has also been analyzed for large velocities and found to have an exponential form $[18,19]$.

\section{ENERGY FLUCTUATIONS IN THE HCS}

To study the effect of velocity fluctuations on the behavior of the total energy of the system in the HCS, we introduce a marginal velocity correlation function by

$$
\varphi_{\mathrm{HCS}}\left(\boldsymbol{c}_{1}, \boldsymbol{c}_{2}\right) \equiv \int d \boldsymbol{l}_{12} \tilde{h}_{\mathrm{HCS}}\left(\boldsymbol{l}_{12}, \boldsymbol{c}_{1} ; \boldsymbol{c}_{2}\right)
$$

An equation for this distribution is obtained by integrating Eq. (53),

$$
\left[\Lambda\left(c_{1}\right)+\Lambda\left(c_{2}\right)\right] \varphi_{\mathrm{HCS}}\left(c_{1}, c_{2}\right)=-\widetilde{\Gamma}\left(c_{1}, c_{2}\right) .
$$

From the definition of $g_{2}$ in Eq. (20) and the scaling of the several distributions defined in the previous section it follows that

$$
\int d c_{1} \varphi_{\mathrm{HCS}}\left(c_{1}, c_{2}\right)=\int d c_{2} \varphi_{\mathrm{HCS}}\left(c_{1}, c_{2}\right)=0 .
$$

Instead of trying to find the complete solution of Eq. (61), we will restrict ourselves to compute what we will call the hydrodynamic part of $\varphi_{\mathrm{HCS}}\left(\boldsymbol{c}_{1}, \boldsymbol{c}_{2}\right)$, defined as follows. We consider the eigenvalue problem associated with the linearized homogeneous Boltzmann operator $[7,8]$

$$
\Lambda(\boldsymbol{c}) \xi_{i}(\boldsymbol{c})=\lambda_{i} \xi_{i}(\boldsymbol{c}) .
$$

The hydrodynamic part of the spectrum of $\Lambda$ is defined by those eigenvalues that coincide with the eigenvalues of the balance equations for the number density, momentum, and temperature following from the homogeneous linearized Boltzmann equation. Such eigenvalues are

$$
\lambda_{1}=0, \quad \lambda_{2}=\frac{\zeta_{0}}{2}, \quad \lambda_{3}=-\frac{\zeta_{0}}{2} .
$$

The corresponding eigenfunctions of $\Lambda$ for each of the above eigenvalues are found to be $[7,8]$

$$
\begin{gathered}
\xi_{1}(\boldsymbol{c})=\chi_{\mathrm{HCS}}(\boldsymbol{c})+\frac{\partial}{\partial \boldsymbol{c}} \cdot\left[\boldsymbol{c} \chi_{\mathrm{HCS}}(\boldsymbol{c})\right] \\
\xi_{2}(\boldsymbol{c})=-\frac{\partial \chi_{\mathrm{HCS}}(\boldsymbol{c})}{\partial \boldsymbol{c}}, \quad \xi_{3}(\boldsymbol{c})=-\frac{\partial}{\partial \boldsymbol{c}} \cdot\left[\boldsymbol{c} \chi_{\mathrm{HCS}}(\boldsymbol{c})\right],
\end{gathered}
$$

respectively. The eigenvalue $\lambda_{2}$ is, therefore, $d$-fold degenerated. The scalar product of two functions $f(\boldsymbol{c})$ and $g(\boldsymbol{c})$ is defined as

$$
\langle f \mid g\rangle \equiv \int d \boldsymbol{c} \chi_{\mathrm{HCS}}^{-1}(\boldsymbol{c}) f^{*}(\boldsymbol{c}) g(\boldsymbol{c}),
$$

with $f^{*}$ being the complex conjugate of $f$. The eigenfunctions $\xi_{\beta}$ given in Eq. (65) are not orthogonal, as a consequence of the operator $\Lambda$ being non-Hermitian in the associate Hilbert space. On the other hand, it is easily verified that the set of functions

$$
\begin{gathered}
\bar{\xi}_{1}(\boldsymbol{c})=\chi_{\mathrm{HCS}}(\boldsymbol{c}), \quad \overline{\boldsymbol{\xi}}_{2}(\boldsymbol{c})=\boldsymbol{c} \chi_{\mathrm{HCS}}(\boldsymbol{c}), \\
\bar{\xi}_{3}(\boldsymbol{c})=\left(\frac{c^{2}}{d}+\frac{1}{2}\right) \chi_{\mathrm{HCS}}(\boldsymbol{c})
\end{gathered}
$$

verify the biorthogonality condition 


$$
\left\langle\bar{\xi}_{\beta} \mid \xi_{\beta^{\prime}}\right\rangle=\delta_{\beta, \beta^{\prime}},
$$

$\beta, \beta^{\prime}=1,2,3$. The definition of the scalar product given above is trivially generalized to two-velocity functions by

$$
\begin{aligned}
\left\langle f\left(\boldsymbol{c}_{1}, \boldsymbol{c}_{2}\right) \mid g\left(\boldsymbol{c}_{1}, \boldsymbol{c}_{2}\right)\right\rangle \equiv & \int d \boldsymbol{c}_{1} \int d \boldsymbol{c}_{2} \chi_{\mathrm{HCS}}^{-1}\left(\boldsymbol{c}_{1}\right) \chi_{\mathrm{HCS}}^{-1}\left(\boldsymbol{c}_{2}\right) \\
& \times f^{*}\left(\boldsymbol{c}_{1}, \boldsymbol{c}_{2}\right) g\left(\boldsymbol{c}_{1}, \boldsymbol{c}_{2}\right) .
\end{aligned}
$$

Then, a projector operator $\mathcal{P}$ is defined as

$$
\mathcal{P} f\left(\boldsymbol{c}_{1}, \boldsymbol{c}_{2}\right)=\sum_{\beta=1}^{3} \sum_{\beta^{\prime}=1}^{3} \xi_{\beta}\left(\boldsymbol{c}_{1}\right) \xi_{\beta^{\prime}}\left(\boldsymbol{c}_{2}\right)\left\langle\bar{\xi}_{\beta}\left(\boldsymbol{c}_{1}\right) \bar{\xi}_{\beta^{\prime}}\left(\boldsymbol{c}_{2}\right) \mid f\left(\boldsymbol{c}_{1}, \boldsymbol{c}_{2}\right)\right\rangle
$$

and the "hydrodynamic part" of $\varphi_{\mathrm{HCS}}\left(\boldsymbol{c}_{1}, \boldsymbol{c}_{2}\right)$ is by definition

$$
\varphi_{\mathrm{HCS}}^{(h)}\left(c_{1}, c_{2}\right) \equiv \mathcal{P} \varphi_{\mathrm{HCS}}\left(c_{1}, c_{2}\right) .
$$

Next we apply $\mathcal{P}$ on both sides of Eq. (61) and, in order to get a closed equation for $\varphi_{\mathrm{HCS}}^{(h)}$, we make the approximation

$$
\mathcal{P} \Lambda\left(c_{i}\right)=\mathcal{P} \Lambda\left(c_{i}\right) \mathcal{P} .
$$

A theoretical estimation a priori of the accuracy of this approximation would require to know more about the spectrum of $\Lambda(\boldsymbol{c})$ and its adjoint than it is available at present. There- fore, it will be taken here as a working hypothesis to be evaluated later on by comparing the predictions it leads to with the results from particle simulations of the system. In this way, we obtain from Eq. (61)

$$
\begin{aligned}
{\left[\Lambda\left(\boldsymbol{c}_{1}\right)\right.} & \left.+\Lambda\left(\boldsymbol{c}_{2}\right)\right] \varphi_{\mathrm{HCS}}^{(h)}\left(\boldsymbol{c}_{1}, \boldsymbol{c}_{2}\right)=-P \bar{T}_{0}\left(\boldsymbol{c}_{1}, \boldsymbol{c}_{2}\right) \chi_{\mathrm{HCS}}\left(\boldsymbol{c}_{1}\right) \chi_{\mathrm{HCS}}\left(\boldsymbol{c}_{2}\right) \\
& +\left[\Lambda\left(\boldsymbol{c}_{1}\right)+\Lambda\left(\boldsymbol{c}_{2}\right)\right] \mathcal{P} \delta\left(\boldsymbol{c}_{1}-\boldsymbol{c}_{2}\right) \chi_{\mathrm{HCS}}\left(\boldsymbol{c}_{2}\right) .
\end{aligned}
$$

By writing

$$
\varphi_{\mathrm{HCS}}^{(h)}\left(\boldsymbol{c}_{1}, \boldsymbol{c}_{2}\right)=\sum_{\beta, \beta^{\prime}=1}^{3} a_{\beta \beta^{\prime}} \xi_{\beta}\left(\boldsymbol{c}_{1}\right) \xi_{\beta^{\prime}}\left(\boldsymbol{c}_{2}\right),
$$

the above equation can be easily solved for the coefficients $a_{\beta \beta^{\prime}}$. Some details of the calculations are given in the Appendix. The result is:

$$
\varphi_{\mathrm{HCS}}^{(h)}\left(\boldsymbol{c}_{1}, \boldsymbol{c}_{2}\right)=a_{33} \xi_{3}\left(\boldsymbol{c}_{1}\right) \xi_{3}\left(\boldsymbol{c}_{2}\right)
$$

where

$$
a_{33}(\alpha)=\frac{d+1}{2 d}+\frac{d+2}{4 d} a_{2}(\alpha)+b(\alpha) .
$$

In the first Sonine approximation, $a_{2}(\alpha)$ is given by Eq. (58) and

$$
b(\alpha)=\frac{2+d-6 d^{2}-\left(10-15 d+2 d^{2}\right) \alpha-2(2+7 d) \alpha^{2}+2(10-d) \alpha^{3}}{6 d(2 d+1)-2 d(11-2 d) \alpha+12 d \alpha^{2}-12 d \alpha^{3}} .
$$

The structure of the result for $\varphi_{\mathrm{HCS}}$ in Eq. (75) is a consequence of the strict conservation of the number of particles and total momentum. Moreover, in the elastic limit $\alpha \rightarrow 1, a_{2}$ vanishes and $b=-(1+d) / 2 d$, so that $a_{33}$ also vanishes, reflecting that the energy is also strictly conserved in this limit, and there are no velocity correlations. On the other hand, for $\alpha<1$, velocity correlations are induced by the inelasticity of collisions and, as a consequence, fluctuations of the total energy $E=\Sigma_{j}^{N} m V_{j}^{2} / 2$ show up. These fluctuations have an intrinsic dissipative character. To compute their second moment, we start by realizing that the energy can be expressed as

$$
E[\Gamma(t)]=\int d x \frac{m v^{2}}{2} F_{1}(x, t),
$$

with $F_{1}(x, t)$ being the microscopic phase space density defined in Eq. (6). The instantaneous deviations of $E[\Gamma(t)]$ from its average value in the HCS are given by

$$
\delta E(\Gamma, t) \equiv E[\Gamma(t)]-\langle E(t)\rangle_{\mathrm{HCS}},
$$

$$
\langle E(t)\rangle_{\mathrm{HCS}} \equiv \int d \Gamma E(\Gamma) \rho_{\mathrm{HCS}}(\Gamma, t) .
$$

Then, using Eq. (78) it is easily seen that

$$
\begin{aligned}
\left\langle[\delta E(t)]^{2}\right\rangle & =\frac{m^{2}}{4} \int d x_{1} \int d x_{2} v_{1}^{2} v_{2}^{2} h_{1,1, \mathrm{HCS}}\left(x_{1}, t ; x_{2}, t\right) \\
& =N k_{B}^{2} T_{\mathrm{HCS}}^{2}(t) \int d \boldsymbol{c}_{1} \int d \boldsymbol{c}_{2} \boldsymbol{c}_{1}^{2} \boldsymbol{c}_{2}^{2} \varphi_{\mathrm{HCS}}\left(\boldsymbol{c}_{1}, \boldsymbol{c}_{2}\right) \\
& =N k_{B}^{2} T_{\mathrm{HCS}}^{2}(t) d^{2}\left\langle\bar{\xi}_{3}\left(\boldsymbol{c}_{1}\right) \bar{\xi}_{3}\left(\boldsymbol{c}_{2}\right) \mid \varphi_{\mathrm{HCS}}^{(h)}\left(\boldsymbol{c}_{1}, \boldsymbol{c}_{2}\right)\right\rangle \\
& =N k_{B}^{2} T_{\mathrm{HCS}}^{2}(t) e(\alpha),
\end{aligned}
$$

where

$$
e(\alpha)=d^{2} a_{33}(\alpha) .
$$

As expected, this function monotonically increases as the value of $\alpha$ decreases, vanishing in the limit of elastic collisions.

To verify the above theoretical predictions, we have performed Molecular Dynamics (MD) simulations of a freely evolving system of inelastic hard disks, i.e. $d=2$, in a square box of size $L$ with periodic boundary conditions. The event 


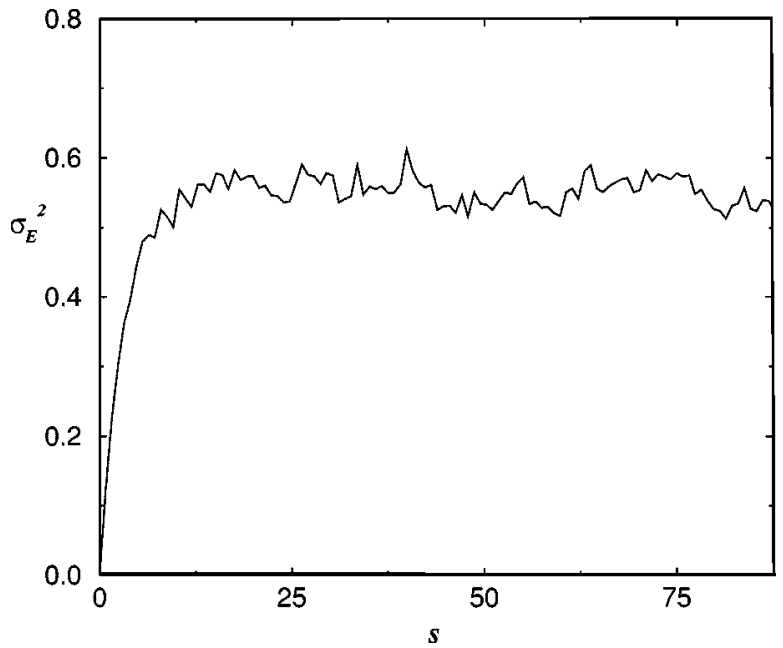

FIG. 1. Scaled dispersion of the energy fluctuations $\sigma_{E}^{2}$ $\equiv N\left\langle(\delta E(s))^{2}\right\rangle /\langle E(s)\rangle^{2}$, as a function of the dimensionless time $s$ defined in the main text, for a system of inelastic hard disks with $\alpha=0.85$ and $n_{H}=0.02 \sigma^{-2}$. The angular brackets average has been taken over 800 trajectories.

driven algorithm [27] has been used. Three different, low values, of the density have been considered, namely $n_{H}$ $=0.02 \sigma^{-2}, 0.01 \sigma^{-2}$, and $5 \times 10^{-3} \sigma^{-2}$. The number of particles used for each of the above densities is $N=1000,2000$, and 4000 , respectively. These values guarantee that, in all the simulations to be reported in the following, the size of the system is smaller than the critical size, $L_{c}$, beyond which the HCS becomes unstable and velocity vortices and spatial inhomogeneities are developed [28]. An estimation of $L_{c}$ has been made by using the hydrodynamic equations obtained from the Boltzmann equation [5,29]. Using this expression, one gets that for the most unfavorable situation to be reported $(\alpha=0.7)$, it is $L / L_{c} \approx 0.86$. Therefore the system is inside the stable homogeneous region. Moreover, we have checked in all the simulations that the system actually stays in the HCS, by monitoring the local velocity and density fluctuations.

For each value of the density and the restitution coefficient a number of trajectories have been generated. By averaging over these trajectories, the time evolution of the average energy and, therefore, of the granular temperature $T_{\text {HCS }}(t)$, has been obtained. In all cases it was very well fitted by Haff's law, Eq. (45). The average value of $E^{2}(t)$ has been obtained in the same way. Afterwards, we have verified that, when the time-dependent energy deviation is scaled with $T_{\mathrm{HCS}}^{2}(t)$, it fluctuates around a stationary average value, as predicted by Eq. (81). An example, corresponding to $\alpha$ $=0.85$ and $n_{H}=0.02$ is shown in Fig. 1. In this case, the results have been averaged over 800 trajectories.

In Fig. 2, the results for the steady values of $\sigma_{E}^{2}$ $\equiv\left\langle(\delta E)^{2}\right\rangle_{\mathrm{HCS}} / N k_{B}^{2} T_{\mathrm{HCS}}^{2}$ are plotted as a function of the coefficient of restitution $\alpha$ for $0.7 \leqslant \alpha \leqslant 1$ for $n_{H}=5 \times 10^{-3} \sigma^{-2}$. Also plotted is the theoretical prediction, i.e., the function $e(\alpha)$ given by Eq. (82). A quite good agreement is observed between the theoretical and numerical results. As expected, the amplitude of the fluctuations monotonically increases as

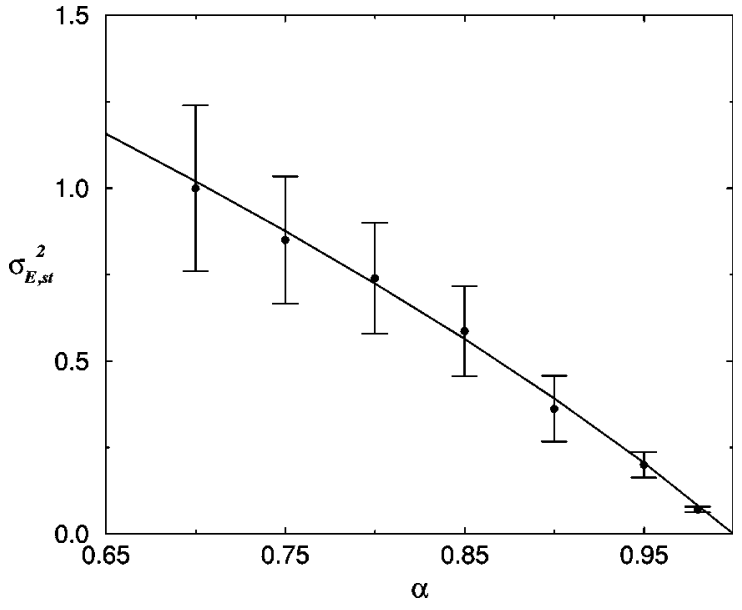

FIG. 2. Average steady values of the scaled second moment of the energy fluctuations $\sigma_{E, \text { st }}^{2}$, as a function of the restitution coefficient. The symbols are from MD simulations of a system of hard disks of density $n_{H}=5 \times 10^{-3} \sigma^{-2}$ while the solid line is the theoretical prediction given in Eq. (82).

$\alpha$ decreases, vanishing in the limit of elastic collisions. The error bars in the figures have been determined from the deviations of the instantaneous values of the scaled energy dispersion from its stationary average value. Similar results have been found for the other two larger densities considered in this paper, although discrepancies between theory and simulations of the order of $10 \%$, probably due to density effects, are observed for $\alpha=0.7$.

We have also investigated the shape of the probability distribution for the energy fluctuations. For each trajectory of the system, the trace of $\left(E(t)-\langle E(t)\rangle_{\mathrm{HCS}}\right) /\langle E(t)\rangle_{\mathrm{HCS}}$ has been partitioned into nonoverlapping bins of value 0.001 and the frequency distribution has been built using the data from all the trajectories corresponding to the same values of $N$ and $\alpha$. The resulting normalized distribution is shown in Fig. 3 for the case $n_{H}=0.01 \sigma^{-2}$ and $\alpha=0.9$, where it is seen that it is very well fitted by a Gaussian distribution (solid line). Simi-

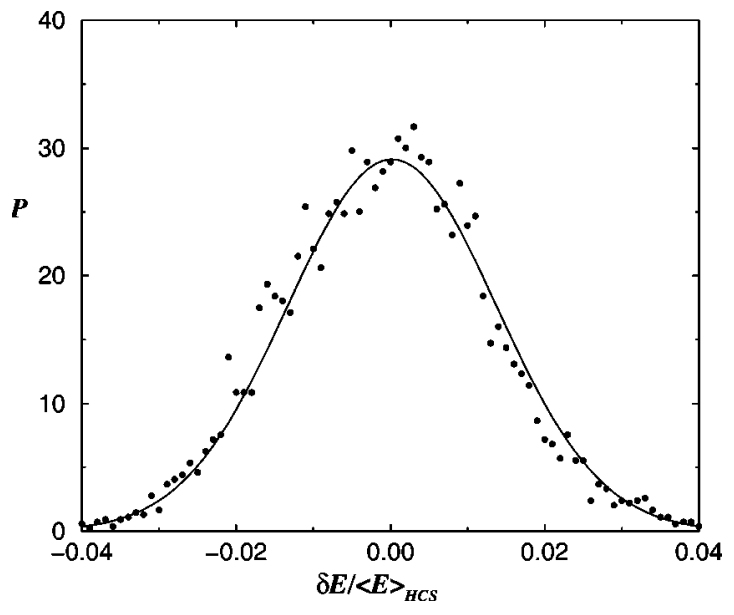

FIG. 3. Normalized distribution of the relative energy fluctuations for a system of inelastic hard disks with $n_{H}=0.01 \sigma^{-2}$. the symbols are from MD simulation and the solid line the fit to a Gaussian. 
lar results are found for the other values of $\alpha$ and $n_{H}$ considered along this work. This suggests the existence of a physical potential governing the amplitude of the energy fluctuations in the HCS, similar to the equilibrium state of ordinary fluids, but we have not been able to identify it.

\section{ENERGY TIME CORRELATION FUNCTION IN THE HCS}

In this section, the time correlation function of the total energy of the HCS, will be studied. To begin with, a global two-time velocity correlation function is introduced by

$$
\psi_{\mathrm{HCS}}\left(\boldsymbol{c}_{1}, s-s^{\prime} ; \boldsymbol{c}_{1}^{\prime}\right) \equiv \int d l_{1} \tilde{h}_{\mathrm{HCS}}\left(\boldsymbol{l}_{1}-\boldsymbol{l}_{1}^{\prime}, \boldsymbol{c}_{1}, s-s^{\prime} ; \boldsymbol{c}_{1}^{\prime}\right),
$$

where $s \geqslant s^{\prime}$. Then, from Eq. (50) it follows that

$$
\left[\frac{\partial}{\partial s}-\Lambda\left(\boldsymbol{c}_{1}\right)\right] \psi_{\mathrm{HCS}}\left(\boldsymbol{c}_{1}, s-s^{\prime} ; \boldsymbol{c}_{1}^{\prime}\right)=0
$$

whose formal solution is

$$
\psi_{\mathrm{HCS}}\left(\boldsymbol{c}_{1}, s-s^{\prime} ; \boldsymbol{c}_{1}^{\prime}\right)=e^{\left(s-s^{\prime}\right) \Lambda\left(\boldsymbol{c}_{1}\right)} \varphi_{\mathrm{HCS}}\left(\boldsymbol{c}_{1}, \boldsymbol{c}_{1}^{\prime}\right) .
$$

As in the previous section, we are interested in the hydrodynamic part of $\psi_{\mathrm{HCS}}$,

$$
\psi_{\mathrm{HCS}}^{(h)}\left(\boldsymbol{c}_{1}, \boldsymbol{c}_{2}\right) \equiv P \psi_{\mathrm{HCS}}\left(\boldsymbol{c}_{1}, \boldsymbol{c}_{2}\right)
$$

Using again the approximation given in Eq. (72), Eq. (85) can be rewritten as

$$
\psi_{\mathrm{HCS}}^{(h)}\left(\boldsymbol{c}_{1}, s-s^{\prime} ; \boldsymbol{c}_{1}^{\prime}\right)=e^{\left(s-s^{\prime}\right) \Lambda\left(\boldsymbol{c}_{1}\right)} \varphi_{\mathrm{HCS}}^{(h)}\left(\boldsymbol{c}_{1}, \boldsymbol{c}_{1}^{\prime}\right)
$$

and substitution of Eq. (75) yields

$$
\psi_{\mathrm{HCS}}^{(h)}\left(\boldsymbol{c}_{1}, s-s^{\prime} ; \boldsymbol{c}_{1}^{\prime}\right)=a_{33} e^{-\left[\zeta_{0}\left(s-s^{\prime}\right) / 2\right]} \xi_{3}\left(\boldsymbol{c}_{1}\right) \xi_{3}\left(\boldsymbol{c}_{1}^{\prime}\right) .
$$

This is our primary result for the spatially integrated hydrodynamic part of the two-time velocity correlation function in the HCS. It shows that the correlation decays exponentially with a rate determined by the cooling rate. In the following, we are going to employ the above expression to compute the HCS time correlation function $C_{E E}\left(t, t^{\prime}\right)$ for the total energy, defined by

$$
C_{E E}\left(t, t^{\prime}\right) \equiv\left\langle E(t) E\left(t^{\prime}\right)\right\rangle_{\mathrm{HCS}}-\langle E(t)\rangle_{\mathrm{HCS}}\left\langle E\left(t^{\prime}\right)\right\rangle_{\mathrm{HCS}}
$$

with $t \geqslant t^{\prime} \geqslant 0$. Here,

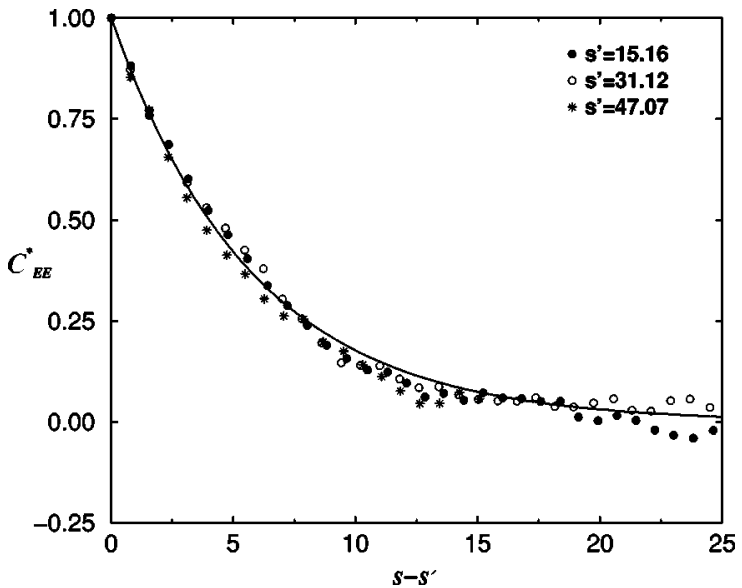

FIG. 4. Normalized energy time autocorrelation function $C_{E E}^{*}\left(s, s^{\prime}\right)$ as a function of $s-s^{\prime}$ for three different values of $s^{\prime}$. The dimensionless time scale $s$ has been defined in the main text. The symbols are from MD simulations of a system of inelastic hard disks with $\alpha=0.85$ and $n_{H}=0.02 \sigma^{-2}$. The solid line is the theoretical prediction as given by Eq. (91).

$$
\left\langle E(t) E\left(t^{\prime}\right)\right\rangle_{\mathrm{HCS}}=\int d \Gamma E[\Gamma(t)] E\left[\Gamma\left(t^{\prime}\right)\right] \rho_{\mathrm{HCS}}(\Gamma, 0) .
$$

Taking into account Eqs. (83) and (51), it is easy to show that the above definition can be transformed into

$$
\begin{aligned}
C_{E E}\left(t, t^{\prime}\right)= & N k_{B}^{2} T_{\mathrm{HCS}}(t) T_{\mathrm{HCS}}\left(t^{\prime}\right) \\
& \times \int d \boldsymbol{c}_{1} \int d \boldsymbol{c}_{1}^{\prime} c_{1}^{2} c_{1}^{\prime 2} \psi_{\mathrm{HCS}}\left(\boldsymbol{c}_{1}, s-s^{\prime} ; \boldsymbol{c}_{1}^{\prime}\right) \\
= & N k_{B}^{2} T_{\mathrm{HCS}}(t) T_{\mathrm{HCS}}\left(t^{\prime}\right) d^{2} \\
& \times\left\langle\bar{\xi}_{3}\left(\boldsymbol{c}_{1}\right) \bar{\xi}_{3}\left(\boldsymbol{c}_{1}^{\prime}\right) \mid \psi_{\mathrm{HCS}}^{(h)}\left(\boldsymbol{c}_{1}, s-s^{\prime} ; \boldsymbol{c}_{1}^{\prime}\right)\right\rangle \\
= & N k_{B}^{2} T_{\mathrm{HCS}}(t) T_{\mathrm{HCS}}\left(t^{\prime}\right) e(\alpha) e^{-\left[\zeta_{0}\left(s-s^{\prime}\right) / 2\right]}
\end{aligned}
$$

This result is consistent with Eq. (81), since the former reduces to the latter for $t=t^{\prime}$ and, therefore, $s=s^{\prime}$.

In Fig. 4 results from MD simulations for a system with $\alpha=0.85$ and $n=0.02 \sigma^{-2}$ are shown. They have been averaged over 800 trajectories. In the figure, $C_{E E}^{*}\left(s, s^{\prime}\right)$ $\equiv C_{E E}\left(t, t^{\prime}\right) / N k_{B}^{2} e(\alpha) T_{\mathrm{HCS}}(t) T_{\mathrm{HCS}}\left(t^{\prime}\right)$ is plotted as a function of $s-s^{\prime}$ for several values of $s^{\prime}$, namely $s^{\prime}=15.16,31.12$, and 47.07. It is seen that the results do not depend on the value of $s^{\prime}$ as implied by Eq. (91). This is a consequence of the scaling property of the HCS, as discussed in Sec. III. Moreover, there is a quite good qualitative and quantitative agreement with the exponential decay predicted by the equation, given by the solid line in the figure and that has been evaluated by using Eq. (59) for the cooling rate $\zeta_{0}$. This is more clearly observed in Fig. 5, where a logarithmic scale has been used. For large times the simulation data become very noisy, but there seems to be some evidence of a systematic deviation from the exponential behavior, becoming stronger as the value of $\alpha$ decreases. Whether this is the case and its possible relationship with hydrodynamic mode coupling effects 


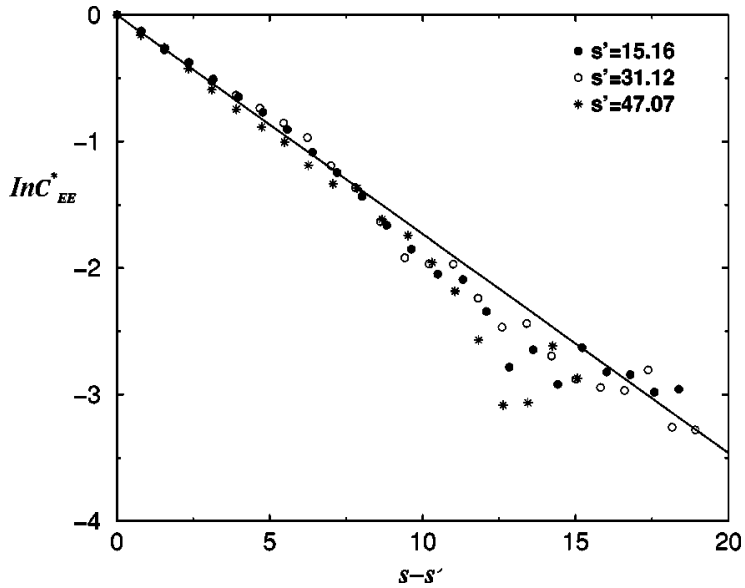

FIG. 5. The same as in Fig. 4 but in a logarithmic plot.

deserves more extensive study. Similar results have been found for other values of the coefficient of restitution in the range $0.7 \leqslant \alpha<1$.

\section{SUMMARY AND DISCUSSION}

In this paper, a general theory for fluctuations and correlations in a dilute granular gas has been formulated, by extending in a natural way the standard methods of kinetic theory. The theory has been particularized for the HCS of a freely evolving granular gas, taking advantage of the scaling properties of this state. Two marginal distribution functions, characterizing the global velocity one-time and two-time correlations, respectively, have been introduced and their hydrodynamic parts have been computed. They are defined by the lowest order eigenfunctions and eigenvalues of the linearized Boltzmann equation, similarly to the case of ordinary fluids with elastic collisions [30,31]. The results, given by Eqs. (75) and (88), show the existence of intrinsic velocity correlations that are induced by the inelasticity of collisions. When properly scaled with the temperature of the system, they decay exponentially with a characteristic time determined by the cooling rate if the time is measured by the average of the accumulated number of collisions per particle. It must be stressed that the existence of these velocity correlations does not imply a violation of the "molecular chaos" assumption that underlies the Boltzmann equation. This is because the latter only refers to the precollisional part of the two-body distribution (at contact) [21]. In fact, it is easily seen that the velocities of two particles are necessarily correlated after they collide, as a consequence of the collision rules. In any case, the simulation results reported in this work show that the correlations are small at least as long as the system is dilute and stays in the HCS.

Let us mention that velocity correlations in the HCS have been recently considered by Pöschel et al. [32]. On the basis of symmetry and simplicity, they assume a form for the reduced two-particle distribution function $f_{2, \mathrm{HCS}}\left(x_{1}, x_{2}, t\right)$ that incorporates both static and dynamic correlations. From this form, it is easy to obtain an expression for the marginal velocity correlation function $\varphi_{\mathrm{HCS}}\left(\boldsymbol{c}_{1}, \boldsymbol{c}_{2}\right)$, introduced in Eq.
(60), by using the definitions given in Sec. II, and afterwards its hydrodynamic part. The functional dependence on the velocity of the particles of the result differs from the one derived here, even in the limit of very small inelasticity. Since no physical foundation is provided by the authors in Ref. [32] for the expression they propose, it is not possible to discuss the origin of the discrepancies.

The one-time and two-time marginal velocity correlation functions determine the fluctuations of the total energy of the system and also its two-time correlation function. Their expressions, given by Eqs. (81) and (91), are two of the main results reported in this paper. To put them in a proper context, it is interesting to analyze the differential equations they obey. Let us first consider $C_{E E}\left(t, t^{\prime}\right)$. By taking time derivative in Eq. (91) and using Haff's law, it is obtained:

$$
\frac{\partial}{\partial t} C_{E E}\left(t, t^{\prime}\right)+\frac{3}{2} \zeta_{\mathrm{HCS}}(t) C_{E E}\left(t, t^{\prime}\right)=0,
$$

valid for $t>t^{\prime} \geqslant 0$. This equation can be given a physical interpretation as follows. Let us write the macroscopic equation for the temperature of the HCS, Eq. (38), in the form

$$
\frac{\partial}{\partial t} E(t)_{\mathrm{HCS}}+M\left[\langle E(t)\rangle_{\mathrm{HCS}}\right]=0,
$$

where $M\left[\langle E(t)\rangle_{\mathrm{HCS}}\right] \equiv \zeta_{\mathrm{HCS}}(t)\langle E(t)\rangle_{\mathrm{HCS}}$. Then, Eq. (92) is equivalent to

$$
\frac{\partial}{\partial t} C_{E E}\left(t, t^{\prime}\right)+L(t) C_{E E}\left(t, t^{\prime}\right)=0,
$$

with

$$
L(t)=\frac{\partial M\left[\langle E(t)\rangle_{\mathrm{HCS}}\right]}{\partial\langle E(t)\rangle_{\mathrm{HCS}}} .
$$

The above relationship between the macroscopic equation for the energy, Eq. (93), and the equation for the time decay of the energy fluctuations, Eq. (94), provides a generalization of the Onsager regression hypothesis for the decay of spontaneous fluctuations in equilibrium, in the sense that the equation describing the dynamics of fluctuations can be obtained from the macroscopic equation describing the evolution of the system.

In a similar way, it is seen that the expression given in Eq. (81) corresponds to the long time limit of the general solution of the equation

$$
\frac{\partial}{\partial t} C_{E E}(t, t)+2 L(t) C_{E E}(t, t)=I(t),
$$

with the source term $I(t)$ given by

$$
I(t)=N e(\alpha) \zeta_{\mathrm{HCS}}(t) k_{B}^{2} T_{\mathrm{HCS}}^{2}(t)
$$

The structure of this equation shows that $I(t)$ can be associated with a "noise amplitude," since it is the average covariance of the noise in an equivalent Langevin formulation [14,35]. In this way, Eqs. (96) and (97) formulate a fluctuation-dissipation theorem for the HCS.

The above results reflect the intuitive notion that the dynamics of low-order correlation functions is essentially of 
macroscopic character. In fact, similar generalizations have been found for many nonequilibrium states of ordinary (elastic) systems by means of different methods and approximations [33,34]. Nevertheless, it must be reminded that the derivation here makes use of the approximation given in Eq. (72).

The theoretical predictions for the fluctuations and the time-correlation function of the total energy of the HCS have been compared with MD simulations, and a good agreement has been found. This provides strong support for the theory developed here, including the hydrodynamic description in terms of the lowest order eigenfunctions and eigenvalues of the linearized Boltzmann collision operator. Of course, the same procedure of analysis can be applied to more involved problems, such as linear response to spatial perturbations, mode coupling effects, and so on.

\section{ACKNOWLEDGMENTS}

We acknowledge economical support from the Ministerio de Ciencia y Tecnología (Spain) through Grant No. BFM2002-00303 (partially financed by FEDER funds).

\section{APPENDIX}

In this appendix we are going to solve Eq. (73) for the coefficients $a_{\beta \beta^{\prime}}$. This is facilitated by using that, for arbitrary functions $f\left(\boldsymbol{c}_{1}, \boldsymbol{c}_{2}\right)$ and $g\left(\boldsymbol{c}_{1}, \boldsymbol{c}_{2}\right)$, it is

$$
\begin{aligned}
& \int d \boldsymbol{c}_{1} \int d \boldsymbol{c}_{2} f\left(\boldsymbol{c}_{1}, \boldsymbol{c}_{2}\right) \bar{T}_{0}\left(\boldsymbol{c}_{1}, \boldsymbol{c}_{2}\right) g\left(\boldsymbol{c}_{1}, \boldsymbol{c}_{2}\right) \\
& =\int d \boldsymbol{c}_{1} \int d \boldsymbol{c}_{2} g\left(\boldsymbol{c}_{1}, \boldsymbol{c}_{2}\right) T_{0}\left(\boldsymbol{c}_{1}, \boldsymbol{c}_{2}\right) f\left(\boldsymbol{c}_{1}, \boldsymbol{c}_{2}\right),
\end{aligned}
$$

where

$$
T_{0}\left(\boldsymbol{c}_{1}, \boldsymbol{c}_{2}\right)=\int d \hat{\boldsymbol{\sigma}} \Theta\left(\boldsymbol{c}_{12} \cdot \hat{\boldsymbol{\sigma}}\right) \boldsymbol{c}_{12} \cdot \hat{\boldsymbol{\sigma}}\left[b_{\boldsymbol{\sigma}}\left(\boldsymbol{c}_{1}, \boldsymbol{c}_{2}\right)-1\right]
$$

The operator $b_{\boldsymbol{\sigma}}$ has been defined in Eq. (5).

Substitution of Eq. (74) into Eq. (73) and use of the linear independence of the functions $\xi_{\beta}$ yields

$$
\begin{aligned}
\left(\lambda_{\beta}+\lambda_{\beta}^{\prime}\right) a_{\beta \beta^{\prime}}= & -\left\langle\bar{\xi}_{\beta}\left(\boldsymbol{c}_{1}\right) \bar{\xi}_{\beta^{\prime}}\left(\boldsymbol{c}_{2}\right) \mid \bar{T}_{0}\left(\boldsymbol{c}_{1}, \boldsymbol{c}_{2}\right) \chi_{\mathrm{HCS}}\left(\boldsymbol{c}_{1}\right) \chi_{\mathrm{HCS}}\left(\boldsymbol{c}_{2}\right)\right\rangle \\
& +\left(\lambda_{\beta}+\lambda_{\beta^{\prime}}\right)\left\langle\bar{\xi}_{\beta}(\boldsymbol{c}) \mid \bar{\xi}_{\beta^{\prime}}(\boldsymbol{c})\right\rangle .
\end{aligned}
$$

For $\beta=\beta^{\prime}=1$ the three terms in the above equation vanish, then becoming an identity. Nevertheless, it is $a_{1}=0$ because of Eq. (62). For $\beta=1$ and $\beta^{\prime}=2$, it is

$$
\begin{aligned}
& \left\langle\bar{\xi}_{1}\left(\boldsymbol{c}_{1}\right) \overline{\boldsymbol{\xi}}_{1}\left(\boldsymbol{c}_{2}\right) \mid \bar{T}_{0}\left(\boldsymbol{c}_{1}, \boldsymbol{c}_{2}\right) \chi_{\mathrm{HCS}}\left(\boldsymbol{c}_{1}\right) \chi_{\mathrm{HCS}}\left(\boldsymbol{c}_{2}\right)\right\rangle \\
& =\int d \boldsymbol{c}_{1} \int d \boldsymbol{c}_{2} \boldsymbol{c}_{2} \bar{T}_{0}\left(\boldsymbol{c}_{1}, \boldsymbol{c}_{2}\right) \chi_{\mathrm{HCS}}\left(\boldsymbol{c}_{1}\right) \chi_{\mathrm{HCS}}\left(\boldsymbol{c}_{2}\right) \\
& =\frac{1}{2} \int d \boldsymbol{c}_{1} \int d \boldsymbol{c}_{2} \chi_{\mathrm{HCS}}\left(\boldsymbol{c}_{1}\right) \chi_{\mathrm{HCS}}\left(\boldsymbol{c}_{2}\right) T_{0}\left(\boldsymbol{c}_{1}, \boldsymbol{c}_{2}\right)\left(\boldsymbol{c}_{1}+\boldsymbol{c}_{2}\right) \\
& =0
\end{aligned}
$$

and $\left\langle\bar{\xi}_{1}(\boldsymbol{c}) \mid \overline{\boldsymbol{\xi}}_{2}(\boldsymbol{c})\right\rangle$ also vanishes because of parity. Then we get $\boldsymbol{a}_{21}=\boldsymbol{a}_{12}=0$.

The remaining coefficients are obtained in a similar way by using Eq. (A1) and the collision rules. The only coefficient different from zero is $a_{33}$ whose expression reads

$$
a_{33}=\frac{\left\langle c^{4}\right\rangle}{d^{2}}+\frac{b_{1}(\alpha)}{\zeta_{0}(\alpha)}+\frac{1}{4}=\frac{d+2}{4 d} a_{2}(\alpha)+\frac{b_{1}(\alpha)}{\zeta_{0}(\alpha)}+\frac{d+1}{2 d},
$$

where

$$
\begin{aligned}
b_{1}(\alpha)= & -\frac{\pi^{(d-1 / 2)}}{\Gamma\left(\frac{d+5}{2}\right) d^{2}} \\
& \times \int d c_{1} \int d c_{2} \chi_{\mathrm{HCS}}\left(\boldsymbol{c}_{1}\right) \chi_{\mathrm{HCS}}\left(\boldsymbol{c}_{2}\right) \vartheta\left(\boldsymbol{c}_{1}, \boldsymbol{c}_{2}\right),
\end{aligned}
$$

with

$$
\begin{aligned}
\vartheta\left(\boldsymbol{c}_{1}, \boldsymbol{c}_{2}\right)= & \frac{\left(1-\alpha^{2}\right)\left(d+1+2 \alpha^{2}\right)}{16} c_{12}^{5} \\
& +\frac{(d+5)-\alpha^{2}(d+1)+4 \alpha}{4} c_{12}^{3} G^{2} \\
& -\frac{1+\alpha}{2}(2 d+3-3 \alpha) c_{12}\left(\mathbf{G} \cdot c_{12}\right)^{2}
\end{aligned}
$$

and

$$
\mathbf{G}=\frac{\boldsymbol{c}_{1}+\boldsymbol{c}_{2}}{2} .
$$

An approximate expression for $b_{1}(\alpha)$ can be obtained by using the result for $\chi_{\mathrm{HCS}}$ in the first Sonine approximation, Eq. (55). In the same approximation $\zeta_{0}$ is given by Eq. (59). In this way, Eq. (75) for $b(\alpha)=b_{1}(\alpha) / \zeta_{0}(\alpha)$ follows.
[1] J. W. Dufty, J. Phys.: Condens. Matter 12, A47 (2000).

[2] I. Goldhirsch, Annu. Rev. Fluid Mech. 35, 267 (2003).

[3] J. J. Brey, J. W. Dufty, and A. Santos, J. Stat. Phys. 87, 1051 (1997).

[4] T. P. C. van Noije, M. H. Ernst, and R. Brito, Physica A 251,
266 (1998).

[5] J. J. Brey, J. W. Dufty, C. S. Kim, and A. Santos, Phys. Rev. E 58, 4638 (1998). Here only the expressions of the transport coefficients for $d=3$ are obtained, while for general $d$ they are reported in Ref. [29]. 
[6] N. Sela and I. Goldhirsch, J. Fluid Mech. 361, 41 (1998).

[7] J. J. Brey, J. W. Dufty, and M. J. Ruiz-Montero, in Granular Gas Dynamics, edited by T. Pöschel and N. Brilliantov (Springer-Verlag, Berlin, 2003).

[8] J. W. Dufty and J. J. Brey, Phys. Rev. E 68, 030302(R) (2003).

[9] J. W. Dufty, A. Baskaran, and L. Zogaib, Phys. Rev. E 69, 051301 (2004); e-print cond-mat/0312113.

[10] J. J. Brey and D. Cubero, in Granular Gases, edited by T. Pöschel and S. Luding (Springer-Verlag, Berlin, 2001).

[11] T. P. C. van Noije, M. H. Ernst, R. Brito, and J. A. G. Orza, Phys. Rev. Lett. 79, 411 (1997).

[12] J. J. Brey, F. Moreno, and M. J. Ruiz-Montero, Phys. Fluids 10, 2965 (1998).

[13] R. Soto, J. Piasecki, and M. Mareschal, Phys. Rev. E 64, 031306 (2001).

[14] M. H. Ernst and E. G. D. Cohen, J. Stat. Phys. 25, 153 (1981).

[15] L. D. Landau and E. M. Lifshitz, Statistical Physics (Pergamon, New York, 1980).

[16] S. R. de Groot and P. Mazur, Nonequilibrium Thermodynamics (North-Holland, Amsterdam, 1962).

[17] A. Goldshtein and M. Shapiro, J. Fluid Mech. 282, 75 (1995).

[18] T. P. C. van Noije and M. E. Ernst, Granular Matter 1, 57 (1998).

[19] S. E. Esipov and T. Pöschel, J. Stat. Phys. 86, 1385 (1997).

[20] J. W. Dufty, Adv. Complex Syst. 4, 397 (2001).

[21] J. Karkheck and G. Stell, Phys. Rev. A 25, 3302 (1982); J. F.
Lutsko, Phys. Rev. Lett. 77, 2225 (1996).

[22] T. P.C. van Noije and M. H. Ernst, in Ref. [10].

[23] J. W. Dufty, M. Lee, and J. J. Brey, Phys. Rev. E 51, 297 (1995).

[24] S. V. Gantsevich, V. L. Gurevich, and R. Katilius, Riv. Nuovo Cimento 2, 1 (1979).

[25] P. K. Haff, J. Fluid Mech. 134, 401 (1983).

[26] J. J. Brey, M. J. Ruiz-Montero, and D. Cubero, Phys. Rev. E 54, 3664 (1996).

[27] M. P. Allen and D. J. Tisdesley, Computer Simulations of Liquids (Oxford Science Publications, New York, 1987).

[28] I. Goldhirsch and G. Zanetti, Phys. Rev. Lett. 70, 1619 (1993).

[29] J. J. Brey and M. J. Ruiz-Montero, Phys. Rev. E 69, 011305 (2004).

[30] P. Résibois and M. de Leener, Classical Kinetic Theory of Fluids (Wiley, New York, 1977).

[31] J. A. McLennan, Introduction to Nonequilibrium Statistical Mechanics (Prentice-Hall, Englewood Cliffs, 1989).

[32] T. Pöschel, N. V. Brilliantov, and T. Schwager, Int. J. Mod. Phys. C 13, 1263 (2002).

[33] A. M. Tremblay, in Recent Developments in Nonequilibrium Thermodynamics, edited by J. Casas-Vázquez, D. Jou, and G. Lebon (Springer-Verlag, Berlin, 1984).

[34] J. W. Dufty, J. J. Brey, and M. C. Marchetti, Phys. Rev. A 33, 4307 (1986).

[35] M. Lax, Rev. Mod. Phys. 38, 359 (1966). 